\title{
Las estrategias de mercado del ecosistema de gestión de las artes plásticas y visuales en Medellín
}

\section{Artículo de investigación}

\section{Laura Rojas De Francisco}

Universidad EAFIT

Irojas3@eafit.edu.co

\section{Carolina García Franco}

Universidad EAFIT

cgarci11@eafit.edu.co

\section{Anny Uribe Saldarriaga Mg}

Universidad EAFIT

auribesa@eafit.edu.co

https://orcid.org/0000-0002-7919-800X

Recibido el: 29/09/2020

Aceptado el: 09/02/2021

Cómo citar este artículo: Rojas de Francisco, L. García Franco, C. y Uribe Saldarriaga Anny (2021). Las estrategias de mercado del ecosistema de gestión de las artes plásticas y visuales en Medellín. Calle 14: revista de investigación en el campo del arte. 16(30), pp.312-329.https://doi.org/10.14483/21450706.18303

\section{(a) (1)}

https://creativecommons.org/licenses/by/4.0/deed.es 


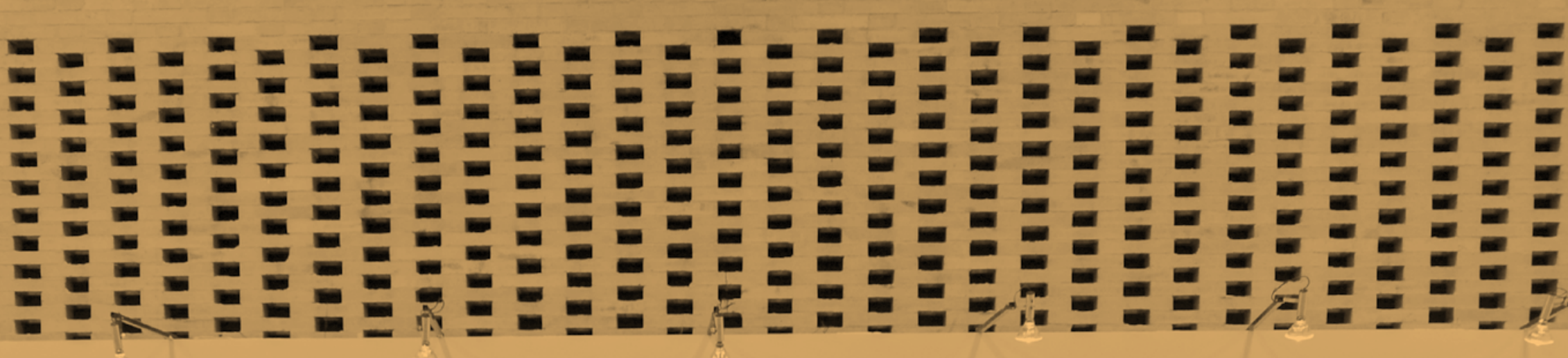

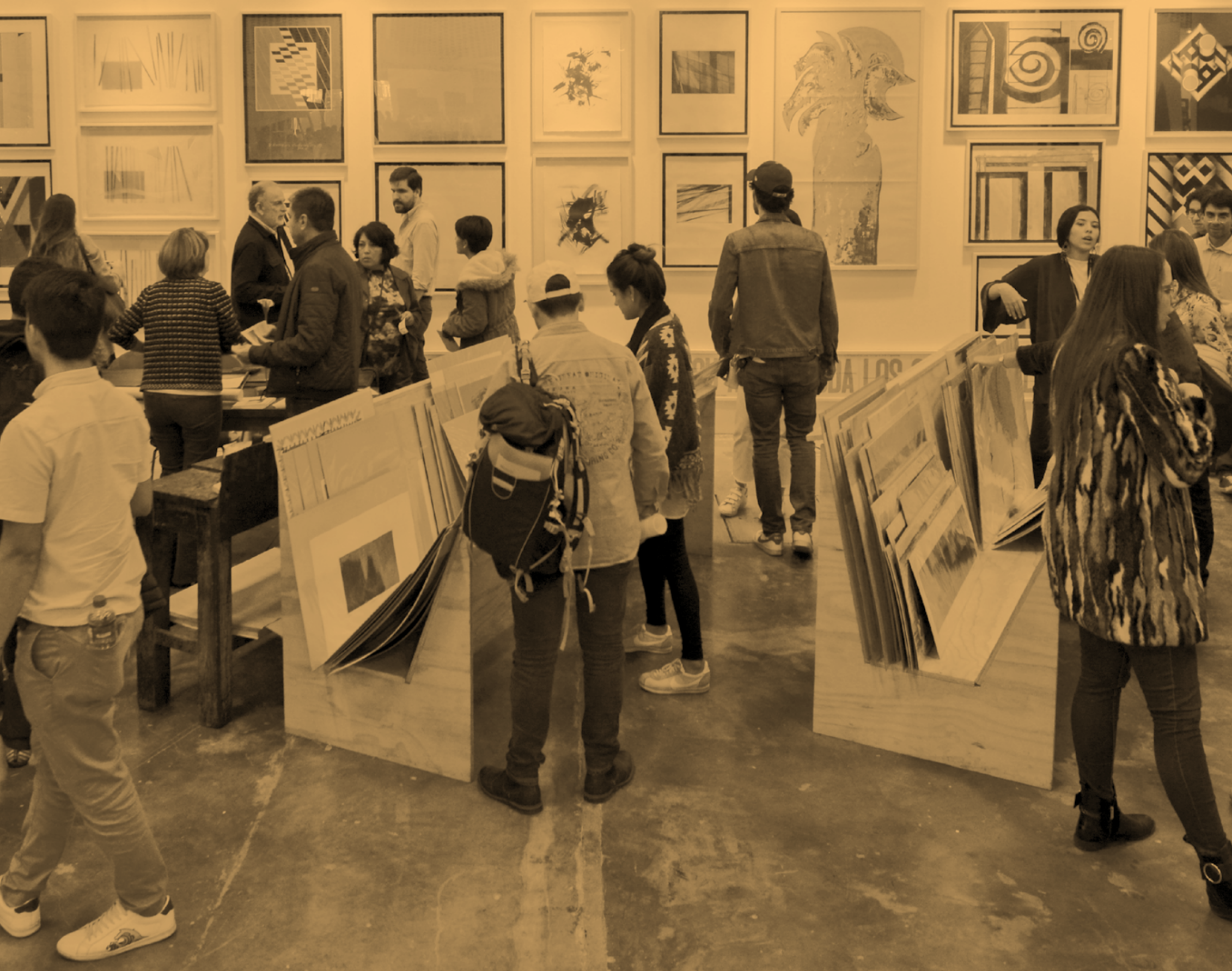


Las estrategias de mercado del ecosistema de gestión de las artes plásticas y visuales en Medellín

\title{
Resumen
}

Las artes plásticas y visuales son parte de las industrias culturales y creativas, y, como tal, requieren esfuerzos para su fomento y promoción. Este estudio identifica prácticas e iniciativas del sector en Medellín para describirlas con una visión ecosistémica, de la mano de una investigación cualitativa que integra revisión de noticias online, políticas públicas, detección de actores del sector cultural y entrevistas que permiten interpretar las acciones, experiencias y relaciones estratégicas que se dan al interior del ecosistema. Los hallazgos permiten visualizar una serie de interacciones que evidencian un ecosistema de gestión y promoción de las artes plásticas en la ciudad, los roles de sus componentes, las acciones hacia la producción cultural y las estrategias de marketing actuales y posibles que pueden aplicarse en otros contextos.

\section{Palabras claves}

Industrias culturales y creativas; promoción cultural; artes plásticas; gestión cultural; economía creativa

\section{Market strategies of the plastic and visual arts management ecosystem in Medellín}

\begin{abstract}
The plastic and visual arts are part of the cultural and creative industries, and, as such, require efforts for their growth and promotion. This study identifies practices and initiatives of the sector in Medellín to describe them with an ecosystems vision, hand in hand with a qualitative research that integrates review of online news, public policies, detection of actors in the cultural sector and interviews that foster the interpretation of the actions, experiences and strategic relationships that occur within the ecosystem. The findings allow us to visualize a series of interactions that show an ecosystem for the management and promotion of the visual arts in the city, the roles of its components, the actions towards cultural production, and current and possible marketing strategies that can be applied in other contexts.
\end{abstract}

\section{Keywords}

Cultural and creative industries; cultural promotion; plastic arts; Cultural Management; creative economy

\section{Résumé}

Les arts plastiques et visuels font partie des industries culturelles et créatives et, à ce titre, requièrent des efforts pour leur croissance et leur promotion. Cette étude identifie les pratiques et les initiatives du secteur à Medellín pour les décrire avec une vision écosystémique, de pair avec une recherche qualitative qui intègre la revue de l'actualité en ligne, les politiques publiques, la détection des acteurs du secteur culturel et des entretiens qui favorisent l'interprétation des actions, les expériences et les relations stratégiques qui se produisent au sein de l'écosystème. Les résultats nous permettent de visualiser une série d'interactions qui montrent un écosystème pour la gestion et la promotion des arts visuels dans la ville, les rôles de ses composants, les actions vers la production culturelle, et les stratégies de marketing actuelles et possibles qui peuvent être appliquées dans d'autres contextes.

\section{Mots clés}

Industries culturelles et créatives ; promotion culturelle ; Arts plastiques ; Gestion culturelle ; économie créative 
As estratégias de mercado do ecossistema de gestão das artes plásticas e visuais em Medellín

\section{Resumo}

As artes plásticas e visuais são parte das industrias culturais e criativas que, como tal, requerem esforços para seu fomento e promoção. Este estudo, identifica práticas e iniciativas do setor em Medellín para descrevê-las como ecossistema mediante a investigação qualitativa, integra revisão de notícias online e políticas, detecta atores do setor cultural e realiza entrevistas que permitem interpretar as ações, experiências e relações estratégicas que se dão ao interior do ecossistema. As evidências permitem visualizar uma série de interações que se evidenciam como um ecossistema de gestão e promoção das artes plásticas na cidade, os papéis de seus componentes, as ações voltadas para a produção cultural e as estratégias de marketing atuais e possíveis que podem ser aplicadas em outros contextos.

\section{Palavras chave}

industrias culturais e criativas; promoção cultural; artes plásticas; gestão cultural; economia criativa

\section{Imasa katungapa pangapi suma ruraskata kawachingapa Medellin sutimanda}

\section{Maillallachiska}

Kaipi Munanaku ningapa Nukanchipa atun Llagtapi tiami llapa suma ruraikuna runakuna pangapi churaska chimanda munankuna kanchasinama kawachinga katungapa. Kunan punchapi tiami llapa kawachidur chipi mana pa churanchi tukuikuna kawachingapa Otka katungapa, ñami iachanchi nigpi tukuikuna chasa rurasunchi mana katima churachukuna.

\section{Rimangapa Ministidukuna}

Maipi ruradiru; kausaita katichidur; kulki maskadur 


\section{Introducción}

La creatividad y la cultura son factores de desarrollo económico; para entidades como UNESCO (2009), OEI, (2019) y UNCTAD (2014), las industrias creativas y culturales - ICC, además de fomentar la cultura, se vinculan con las economías del conocimiento donde la creatividad es un recurso que añade valor a bienes materiales e intangibles (Boix Domenech y Lazzeretti, 2012). Por esto, hay que promoverlas con estrategias de comunicación, lo que parte de conocer las necesidades e intereses de consumo cultural para lograr las formas adecuadas para promocionarse (Boorsma, 2006).

En ese panorama, entender la oferta cultural que se da en las ciudades es una oportunidad de estudio y en este artículo se analiza el mercado de las artes plásticas y visuales en la ciudad de Medellín, para identificar cómo se desarrollan las estrategias de gestión y comercialización del sector cultural; entendiendo la estrategia como el compilado de procesos, que incluyen planes y acciones de marketing, en este caso, ajustadas al carácter de las ICC (Kolb, 2013). A la vez, se concibe que hay una serie de dinámicas que funcionan como un conjunto de relaciones y articulaciones como parte de un ecosistema (sistema vivo), con dinámicas en permanente transformación, que responde a las lógicas, las demandas y las ofertas del contexto, de los sectores que la conforman y de los actores que intervienen (i.e. artistas, galerías, museos, etc.). Por lo que se hace seguimiento a estrategias de promoción y divulgación en un tiempo y espacio determinados.

Se recurre a una metodología cualitativa, con análisis de publicaciones sobre el tema cultural, que permiten identificar actores, acciones e iniciativas de promoción y gestión de este ecosistema y desarrolla un trabajo de campo con actores detectados, para exponer las estrategias de mercado y recoger la visión de conocedores del tema en la ciudad, para identificar estrategias de marketing, productos, tipos de consumidores y peculiaridades del mercado de las artes plásticas y visuales de la ciudad de Medellín que puedan orientar acciones hacia fortalecer o facilitar el consumo y disfrute de los productos del sector.

\section{Revisión de la literatura}

La cultura en un sentido amplio, es considerada por UNESCO (2012), como el conjunto de rasgos distintivos, espirituales, materiales, intelectuales y afectivos que caracterizan a una sociedad o un grupo social. Como actividad económica, el sector de las industrias culturales y creativas, comprende actividades de creación, producción y distribución de bienes y servicios en diversas áreas, dentro de las cuales, están las artes plásticas y visuales, aquellas que utilizan materiales que pueden ser modificados o moldeados por artistas para crear una obra producto de su imaginación o su visión de la realidad, e incluyen pintura, escultura, dibujo, arquitectura, grabado, cerámica, muralismo, o expresiones que incorporan tecnologías y pueden relacionarse con otras disciplinas artísticas por vínculo creativo o su expresión. Además, como patrimonio material o intangible, son un bien común que beneficia a las personas, e integra identidades personales, comunitarias y territoriales (Silverman \& Fairchild-Ruggles, 2007).

Las ICC como sector económico, tienen un rol con diversidad de lógicas, políticas, contextos y sistemas de producción, donde la obra artística con su sentido único y particular, se convierte en producto para el consumo y responde a una demanda (Miller, 2009). Así, cualquier persona con interés en sus productos y servicios, compra y puede convertirse incluso en coleccionista o invertir en arte (O'Connor, 2000; Vattese, 2002), por eso, se manejan precios de mercado y se consideran tendencias artísticas, que se proyectan hacia las preferencias del consumo (Pérez-Calero Sánchez et al., 2011). También hay consumo itinerante cuando se invierte en vivir la experiencia con la obra y los artistas, como sucede en los museos (Galí-Espelt, MajóFernández y Vidal-Casellas, 2000).

En ese panorama, la gestión cultural, puede generar oportunidades de desarrollo, a partir de reconocer en el contexto, las prácticas, acciones y las sinergias para posicionar las creaciones culturales en escenarios de intermediación, negociación o promoción (Gomez, 2006), que requieren gestionar acciones para facilitar, promover, estimular, conservar y difundir las diferentes actividades y manifestaciones culturales (CatalánRomero y González-Rueda, 2014). Es en este proceso, que entra el marketing cultural, donde las prácticas de comercialización, integran intermediarios como galeristas, subastadores y agentes que ponen a disposición de consumo o exhibición las creaciones que relacionan a creadores y artistas, con coleccionistas, audiencias 0 espectadores (Vesga, 2005).

El producto artístico es único, satisface a quienes tienen gusto o vinculación con la obra o el artista (Boorsma \& Chiaravalloti, 2010), puede expresar 
emociones e ideales estéticos y eso le diferencia, pues es una transacción, que comienza con los mismos creadores y artistas, - sus consumidores iniciales-y luego, hay intercambios con quienes se identifican con la visión de estos primeros (López-Sintas, 2015). Por esto, para pensar un mercado de consumidores de experiencias artísticas, una estrategia puede ser la de identificar atributos que hacen posible su disfrute (i.e la visión de creadores) y luego sí dirigirse a un segmento de consumidores o usuarios de un consumo relacionado con la experiencia cultural que pueda responder a esos atributos (Kolb, 2013).

En el mercado del arte, los segmentos pueden establecerse según las motivaciones de consumidores, en este sentido Boorsma y Chiaravalloti (2010) plantean una visión desde la experiencia, donde los consumidores tienen un rol que incide en el desempeño del mercado artístico, por lo que se requiere encontrar valores de la experiencia de consumo en la creación y recepción de la obra, para una segmentación desde las experiencias, preferencias y actividades de ocio, que pueden ser de consumo omnívoro (Fernández \& Heikkilä, 2011), un consumo que puede darse como actividad de ocio - cultural- que puede incluir tanto lo considerado como popular o de élite o ser para consumidores que compran o públicos que son espectadores de las obras (Gürel \& Nielsen, 2019; Kolb, 2013).

Por lo tanto, de lo que trata el marketing cultural, es de la estrategia de búsqueda para identificar y alcanzar segmentos de mercado, donde se ajusta la mezcla de marketing al producto del proceso creativo, al bien cultural (Azuela Flores et al., 2010). Esto se haría señalando al producto, localizando consumidores y eligiendo el mercado; buscando equilibrio entre lo que son las necesidades de consumo y lo que es un proceso de mercado y la integridad artística, esto es, preservando las características del proceso y la idea de la creación, que estratégicamente es lo que le da al valor agregado (Mollá-Descals y Cuadrado-García, 2000).

Posicionar un bien cultural o artístico, permite a gestores y organizaciones culturales alcanzar segmentos del mercado interesados, e identificar deseos y necesidades de audiencias y consumidores en un proceso social de intercambio que supone crear, comunicar, promocionar, entregar valor y gestionar relaciones; beneficiando a creadores, organizaciones, grupos de interés y sociedad (Pérez y Sánchez, 2011).
Para eso se requieren políticas culturales de soporte, en Colombia, la ley de fomento a la economía creativa busca desarrollar, fomentar, incentivar y proteger las ICC, y generar valor, sobre la propiedad intelectual, origen y alcance cultural, con una perspectiva multisectorial (Ley Naranja, 2017), y con el decreto para la creación del Consejo Nacional de la Economía Naranja, (2018), se promueven actividades económicas y exportación de productos de las ICC, da bases de financiación, incentivos fiscales e implementación para el mecenazgo (Ley de Financiamiento, 2018).

En Medellín se cuenta con la política pública de Museos del Municipio de Medellín, (2015) y la política del Concejo de Medellín (2016), hechas para consolidar y sostener las instituciones y programas del campo, lograr reconocimiento y valoración de la riqueza cultural, fortalecer la capacidad institucional de museos y propiciar la operación eficiente en la gestión del patrimonio, la formación de públicos y la sostenibilidad intersectorial; así como fomentar las capacidades de gestión y acciones financieras, con estrategias de acceso al patrimonio y la cultura y un sistema de información e indicadores del sector.

\section{Metodología}

Este artículo, mediante un estudio cualitativo interpretativo-hermenéutico (Guba y Lincoln, 1994), identifica las estrategias de gestión cultural y marketing y caracteriza el ecosistema del sector de las artes plásticas en la ciudad de Medellín. Para hacerlo, se recurrió a la recopilación de documentos públicos y publicaciones para detectar las fuentes primarias e identificar: 1) actividades de gestión y producción cultural, 2) actores o personas y entidades que gestionan la cultura y 3) acciones de comercialización que se conforman como posibles estrategias.

La información recopilada de repositorios históricos de noticias de cinco años (157), una vez filtrada llevó a 67 analizadas siguiendo los objetivos (listado disponible bajo requerimiento). Los perfiles detectados llevaron a la realización de entrevistas semi estructuradas con duración promedio de 60 minutos, grabadas con autorización firmada a diez personas expertas y referidas entre artistas y creadores, curadores, galeristas o promotores, gestores culturales, periodistas culturales, compradores de arte y públicos. El protocolo se compone de bloques temáticos que se constituyen como categorías, estas son: gestión cultural, ecosistema de 
creación y comercialización, estrategias y acciones de promoción y oportunidades.

El análisis se hizo con matrices por objetivos y categorías de análisis, que integran tanto las noticias tratadas con procedimientos de hemerografía (Páez, Atencio y Neüman, 2013), como las entrevistas, donde se valoran aportes temáticos o categorías emergentes, que permiten construir una narrativa recurriendo a la descripción densa (Geertz, 2003), para establecer características y relaciones entre actores del ámbito cultural de las artes plásticas y visuales que componen el ecosistema (González-Vélez, 2015), la dinámica del sector y las problemáticas y oportunidades de acción estratégica para su mercado.

\section{Hallazgos}

El ecosistema de artes plásticas y visuales de Medellín estudiados en este artículo, integra componentes y roles estratégicos del mercado, actores con funciones propias del mercado y da cuenta de las estrategias para dar a conocer obras y creadores, así como las dinámicas y relaciones con consumidores y públicos, la intermediación y la toma de decisiones de comercialización. A continuación, se describen e ilustran:

\section{Actores y acciones del ecosistema de artes plásticas y visuales}

El rol de la academia, va más allá de la formación, es dónde la promoción del arte comienza, Constanza Toro pone como ejemplo la Universidad Nacional de Medellín, pionera por su plan estratégico para promover el arte del Taller Central, que considera propuestas artísticas de jóvenes promesas; aunque expone que falta difusión para lograr que las obras lleguen más allá de eventos especiales, para convocar y estimular públicos. Quienes participaron en las entrevistas coinciden apuntando a la necesidad de dar bases para que artistas y creadores sepan cómo presentar propuestas y obtener presupuesto.

Por otro lado, tanto publicaciones como entrevistados coinciden en la necesidad de trabajar en formación de públicos, donde la política pública incluya el tema, en planes estratégicos locales y nacionales y superar la idea del sector cultural como no generador de beneficios económicos y al que se le recorta presupuesto. Hay quien refiere como ejemplo de inversión al Museo de Arte Moderno - MAMM y el efecto en la zona que influencia, identificada como espacio para la agenda cultural y turística.

Según algunos de los expertos entrevistados, el rol de la curaduría para organizar obras y colecciones también sirve para llegar a los públicos, pues como mediadores entre el arte y los públicos acercan el arte y su lenguaje a las personas y en especial, pueden ampliar la mirada cuando son institucionales (museos). Pueden actuar de manera autónoma y convertirse en agentes promotores culturales. Según Monica Quintero, periodista cultural, la carrera de curadores es reciente y la academia en Medellín aún no cubre todo, pero ahí hay una conexión estratégica por fortalecer con la academia. Los curadores venden proyectos y comercializan artistas, vuelven protagonistas las obras de las que son interventores. Por su parte, Museos y galerías tienen roles promotores y gestores, pero según la artista Ana Isabel Díez, los museos tienen esquemas curatoriales complejos y comprometen tiempo, mientras las galerías son ágiles.

El rol de las galerías es la intermediación entre artistas y coleccionistas o consumidores de obra de arte, es la estrategia más orientada a mercados. Comienza con contacto directo y por sistema de recomendación, como parte de su estrategia se aseguran de tener buen relacionamiento con colectivos, artistas y curadores, participan en proyectos individuales y colectivos, ofrecen obras a coleccionistas por medios directos y muy poco por medios masivos o digitales. Es de anotar que no tienen relación con entidades públicas, pues destinan los recursos a otras prioridades. Lo anterior se amplía en el gráfico 1.

Los artistas y creadores hacen parte de todo el entramado del ecosistema. Los artistas Belarmino Miranda y Carlos Bermudez, al exponer sus apreciaciones, coinciden en tres posibles estratégias para integrarse al mercado: la primera se presenta cuando han tenido buenas relaciones sociales, que llevan a contactos con el mercado, ahí deben contar con un buen cuerpo de obra que posibilite dirigirse a una galería y ser parte de una red de contactos. Así, cuando hay trabajo conjunto, pueden realizar proyectos con curadores, exhibir donde hay alineación de conceptos, sean exposiciones individuales o colectivas (museos, galerías o sitios públicos). La exhibición, atrae público que conozca la obra y promueve transacciones a través de la galería o directamente.

La segunda estrategia es con curadores que buscan artistas para proyectos y exhibiciones, atrae público 


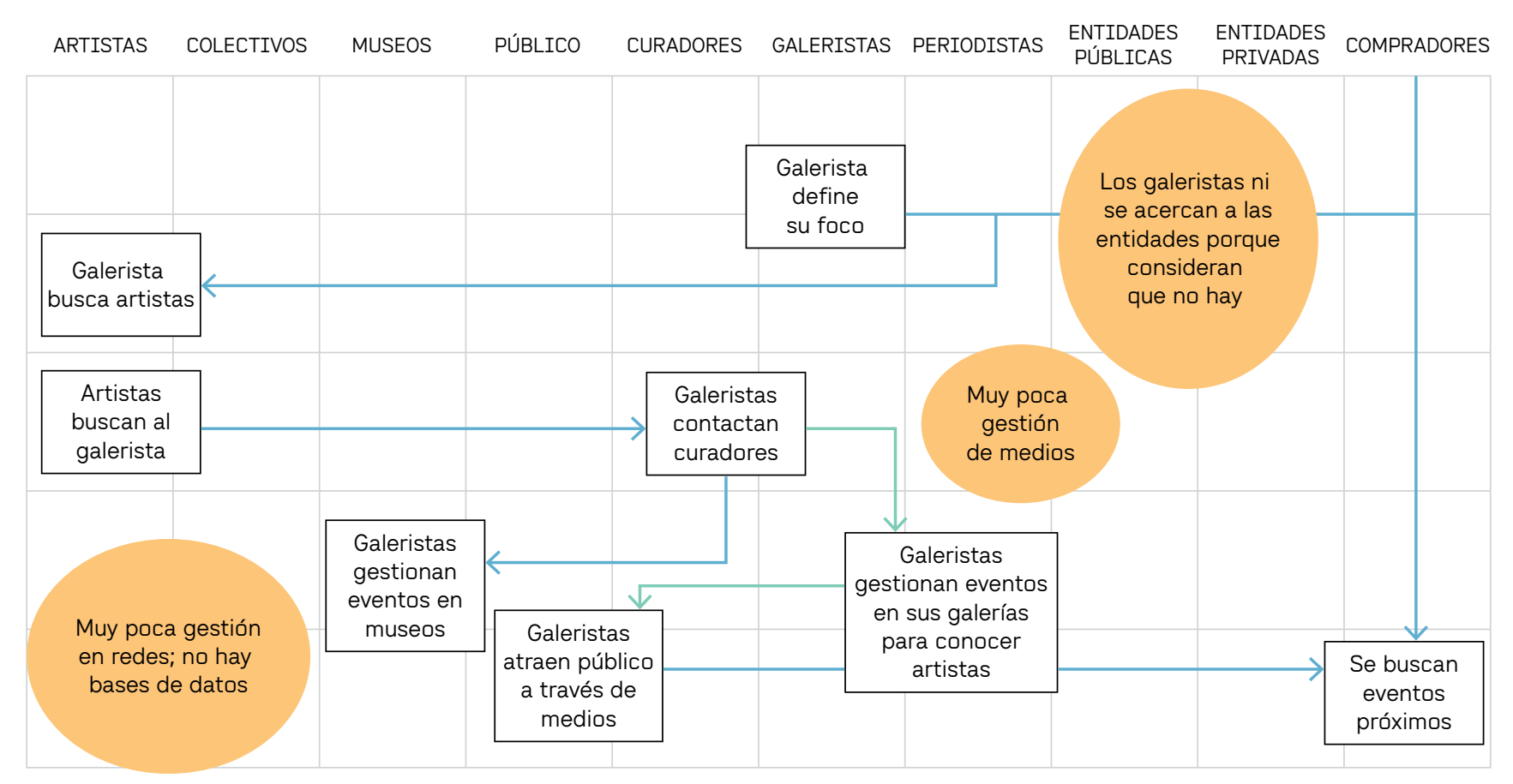

Oportunidades o retos en el proceso

Gráfico 1. Proceso de gestión: Galerías. Elaboración propia.

conocedor y genera negociaciones. La tercera estrategia, es participar en convocatorias del Estado, aunque expresan que a pocos les llega la información o no conocen procedimientos de participación, esto último un asunto relacionado con formación desde la academia. Para estas opciones, deben contar con portafolio o canales digitales para contacto o centralizar información. El gráfico 2, expone el proceso desde la perspectiva de los artistas.

Las evidencias sobre el rol de los colectivos muestran que una vez finalizada la formación, los artistas buscan estratégicamente pertenecer a un colectivo, generar relaciones con otros artistas, participar de proyectos en conjunto y mantener actividad en la construcción de obras. Esto les permite interactuar, compartir conocimientos y experiencias, buscar alternativas de proyectos y participar en eventos apoyados por entidades públicas y privadas (i.e. exposiciones, ferias), llegando a públicos interesados en experimentar o consumir. Con los colectivos la relación es formativa, de desarrollo conceptual y de gestión para llegar a públicos con exposiciones en diversos espacios de la ciudad.

Con las galerías la estrategia es compartir información, para los galeristas, esto requiere contar con bases de datos para localizarlos, o canales de comunicación y promoción, pero los artistas que participaron, mencionan que funciona más el contacto entre referidos, porque esas bases de datos pueden estar desactualizadas. El gráfico 3, muestra las acciones colectivas.

El rol de los públicos es esencial para dar a conocer los productos culturales y generar un gusto por el consumo de artes, por eso es estratégico llegar al público en general, pero lograr interés es difícil cuando este no tiene formación en arte. Las evidencias muestran iniciativas de formación de públicos con eventos de arte en la ciudad, pero algunos entrevistados exponen que muchas veces los públicos asisten por casualidad y sobre todo que prima la gratuidad como costumbre. Sin embargo, esto no deja de ser una oportunidad para formar públicos, pues cuando les gusta, se interesan, buscan información y hay sensibilización que les puede llevar a tener un grado de conocimiento y querer disfrutar de la producción cultural.

Los públicos cuando están formados y conocen del tema, se esfuerzan por conocer sobre lo qué pasa en la ciudad, esto les lleva a conocer artistas y por lo tanto suelen comprar, ya sea para decoración, regalo o colección. según algunos artistas y los galeristas entrevistados, la negociación es sin intermediarios, contactan directamente a los artistas conocidos y recomendados o tienen en mente a los que han sido destacados en eventos y colectivos que siguen en medios. Estos 


$\begin{array}{llll}\text { ARTISTAS COLECTIVOS MUSEOS PÚBLICO CURADORES GALERISTAS PERIODISTAS ENTIDADES } & \text { ENTIDADES COSICAS PRIVADAS COMPRADORES }\end{array}$

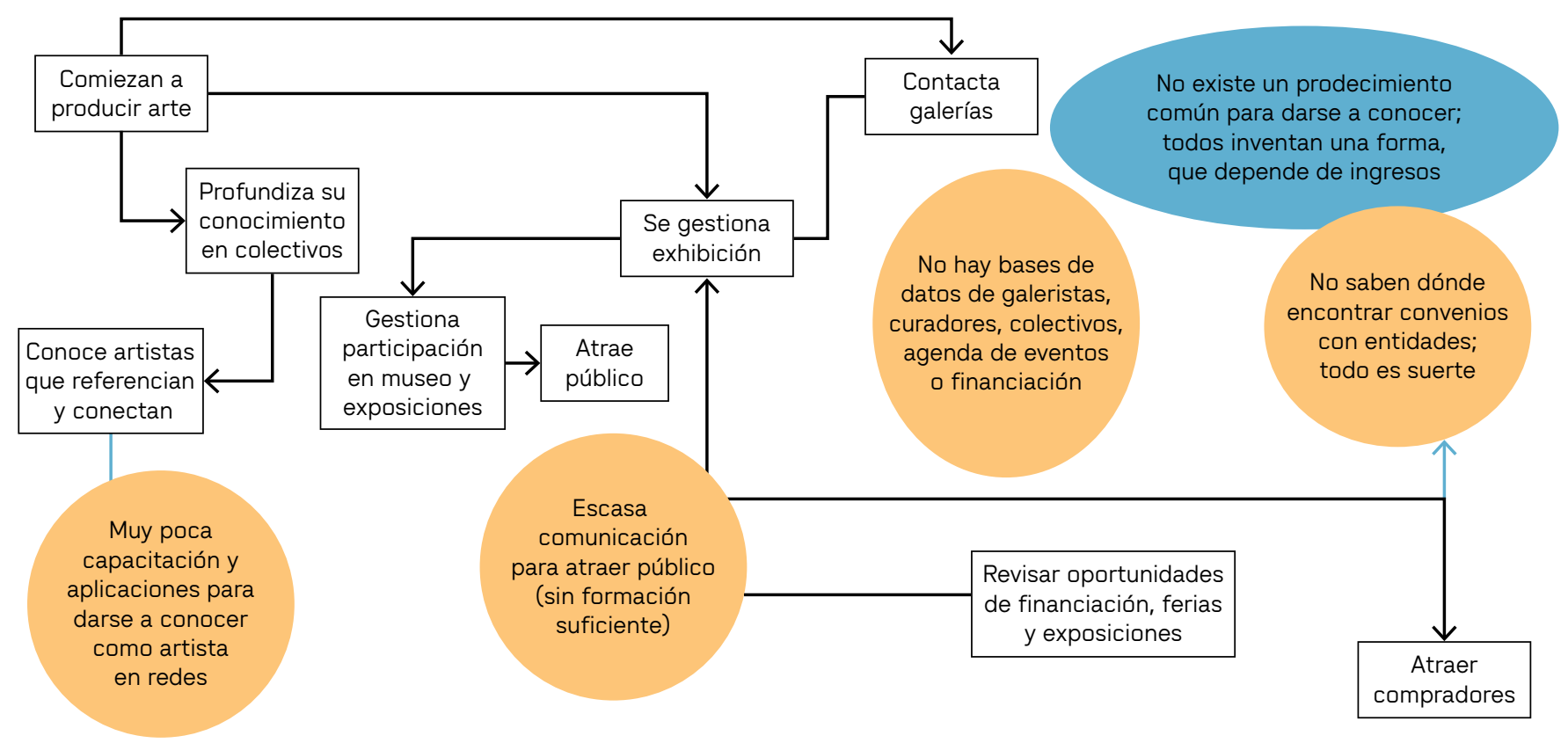

Oportunidades o retos en el proceso

Gráfico 2. Proceso de gestión: Artistas. Elaboración propia

$\begin{array}{llll}\text { ARTISTAS COLECTIVOS MUSEOS PÚBLICO CURADORES GALERISTAS PERIODISTAS ENTIDADES } & \text { ENTIDADES } \\ \text { PÚBLICAS } & \text { PRIVADAS }\end{array}$

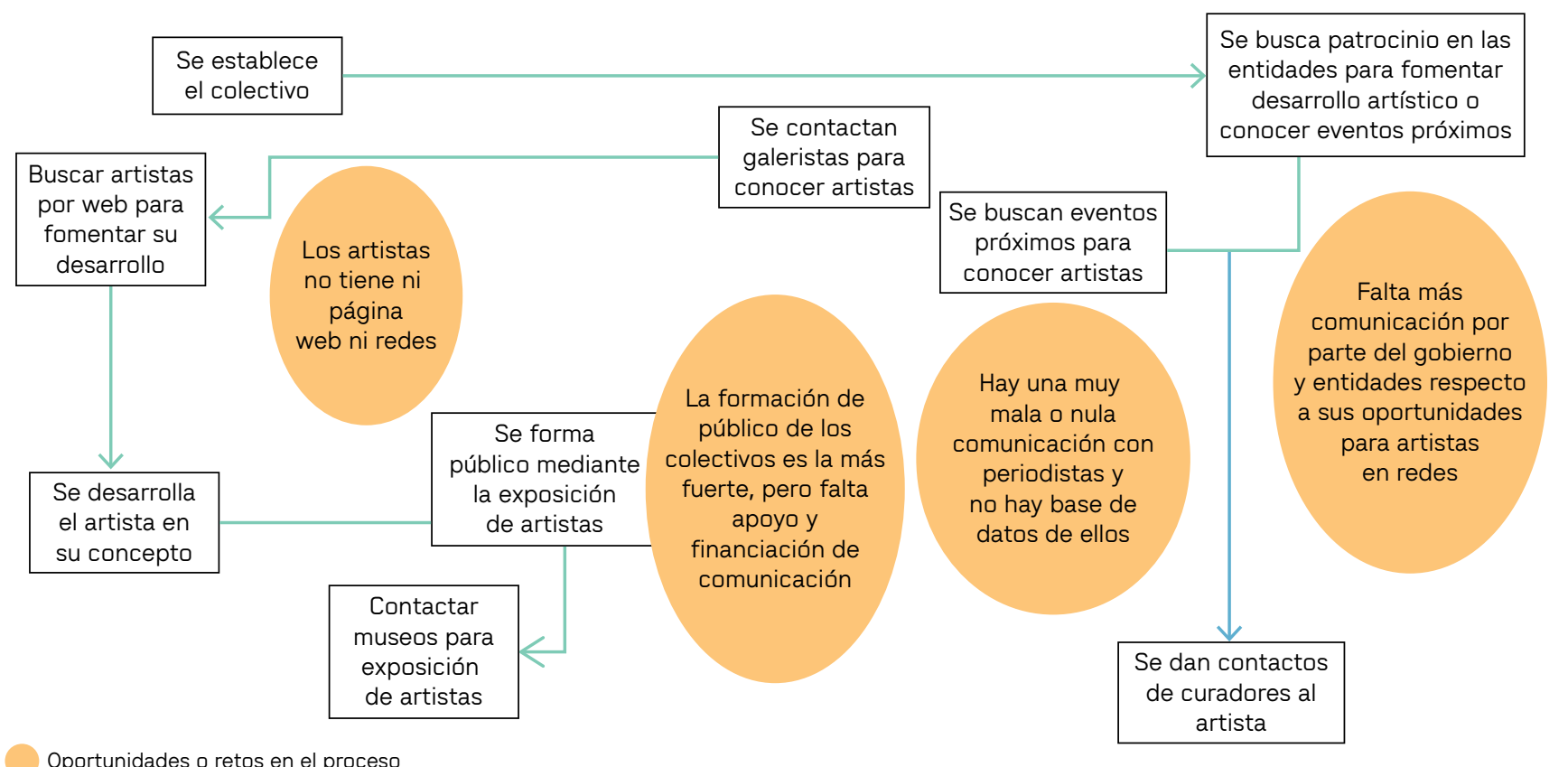

Gráfico 3. Proceso de gestión: Colectivos. Elaboración propia. 


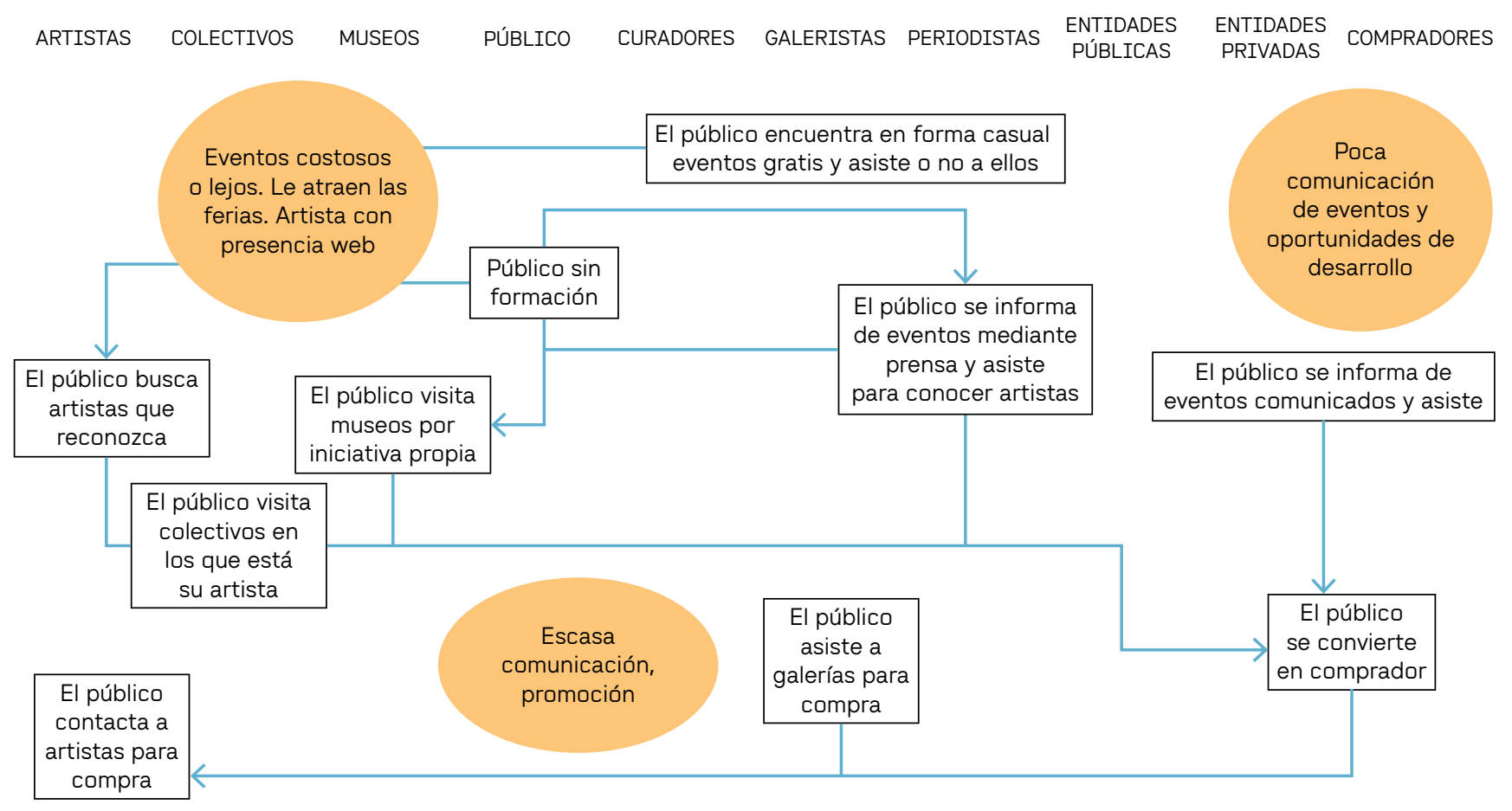

Oportunidades o retos en el proceso

Gráfico 4. Proceso de consumo públicos. Elaboración propia.

públicos consumen por gusto, programan asistir a lo que les interesa y entran en contacto con diferentes experiencias o lugares donde están los artistas, aumentando su conocimiento. El gráfico 4 amplia lo expuesto.

Existen otros actores en el ecosistema, estos comprenden la empresa privada que apoya eventos y programas culturales o la ampliación de instalaciones. También la empresa realiza procesos conjuntos con la Alcaldía, para realizar actividades de formación, proponer espacios o legalizar procesos de comercialización con la Cámara de Comercio; esto por ejemplo se aprecia en Cultura $E^{1}$, un programa de impulso al emprendimiento que incluye iniciativas culturales. También destaca el papel de los medios de comunicación como la columna de cultura del periódico El Colombiano, parte integral de una estrategia de comunicación donde se constituyen canales de información para la agenda cultural. Finalmente, se aprecian los espacios autogestionados que se vienen fortaleciendo, y que, estratégicamente hablando, tienen un rol de integración dentro del ecosistema sobre el que cabe un estudio adicional, porque trascienden las instituciones y cobijan iniciativas independientes, facilitando así tanto la creación y oferta cultural como su consumo.
Con los actores identificados, los hallazgos que apuntan a las acciones estratégicas que vienen sucediendo en la movida artística en Medellín, se ve el impulso de colectivos de creadores y organizaciones culturales que, para Quintero, desarrollan iniciativas que se generan con o sin recursos públicos. Esto conlleva a que las propuestas artísticas se vengan acercando a las personas, la política pública para la cultura, está encaminada a lograr reconocimiento y valoración de la riqueza cultural y el fortalecimiento de la capacidad institucional para organizar y consolidar el sector. Se apunta hacia la operación eficiente en la gestión del patrimonio y a la sostenibilidad intersectorial, con medidas que buscan fortalecer capacidades de gestión y financiera y que se evidencian en iniciativas como la Mesa de Museos que según María del Rosario Escobar, directora del Museo de Antioquia, es un mecanismo de conversación

Santiago Ortiz, presidente de la Red de Museos de Antioquia y director del Museo de la Universidad de Antioquia, explica que la Mesa busca integrar las galerías tradicionales de arte, tanto en contenido, como en espacio; pensar las entidades museísticas como base para el entendimiento mutuo, la cooperación y el enriquecimiento cultural, donde los museos son recurso didáctico, son un apoyo académico abierto a distintos 
públicos, en donde cada sala de arte, tiene exposiciones itinerantes para divulgar patrimonio.

En la formación de públicos, hay estrategias como la del Museo de Antioquia, una institución que se ha posicionado activamente como agente cultural en los procesos de transformación de la ciudad, ha logrado posicionarse como centro cultural interdisciplinario referente, que propende por convertir al museo en escenario de interacción educativa y cultural al convocar a la participación. Iniciativas como "La consentida"2 buscan acercar al público a obras, colecciones y documentos del arte, sumándose, además, en el proceso a la recuperación del centro.

EI MAMM, fomenta las artes plásticas, las audiovisuales, las escénicas y la música, generando una oferta cultural amplia para múltiples públicos. Para su directora, María Mercedes González, la expansión del lugar y la programación, fortalece la oferta turística, cultural y de entretenimiento, permite a la ciudad redescubrirse y reinventarse y proporcionar calidad a las actividades culturales, satisfaciendo los intereses del público con experiencias memorables, divertidas, entretenidas.

En esa relación con públicos las acciones en espacios de la ciudad son estratégicas. Con los Encuentros Internacionales de Medellín - MDE, el Museo de Antioquia, vincula lo urbano y la diversidad, combinando prácticas artísticas y espacios no artísticos, que posicionan la ciudad en escenarios internacionales artísticos. Otra iniciativa es Museo 360, que, para Carlos Uribe, curador del museo, es plataforma de alfabetización ciudadana, activa el espacio público del centro de Medellín.

Por su parte, las galerías tienen una función mercantil, pueden tanto vender a puerta cerrada a coleccionistas e invitados que les aseguran mercado, como acercar el arte al público. Según Pilar Velilla de la Galería Naranjo y Velilla, tienen la labor cultural de orientar a partir de una curaduría documentada que dé conocimiento del mercado del arte. Sobre esa convocatoria que puede hacerse al público, Harold Ortiz, director y fundador de Timebag, plantea que las galerías crean dinámicas y experiencias de arte. En ese sentido por ejemplo se puede exponer una práctica estratégica como la explica Liliana Hernández de Plecto Galería, quien cuenta que la galería no tiene catálogo de obras en existencia, pues trabaja por proyectos colectivos e individuales. Por su parte en Lokkus, una galería de arte contemporáneo, según resalta Manuela Velásquez, nació para promover jóvenes artistas y permitir en distintos ambientes, tener experiencias con deferentes personas e ideas e interactuar, trabajar y exponer. Esto es, estratégicamente funciona como potenciador que además, hace formación de públicos con programas educativos, charlas, visitas guiadas y espacios con puntos de vista espirituales y de conocimiento; además permite a los consumidores acercarse a los artistas en sus talleres, escuchar sobre su proceso y saber el por qué de lo que hacen.

La trayectoria de instituciones como La Oficina o la galería de Julietta Álvarez con colaboración y autogestión, amplían y enriquecen la escena del arte. Las galerías Lokkus, Plecto y La Oficina también han realizado iniciativas como ARTMED y el Día de las Galerías, para unirse y ampliar círculos, según Manuela Velásquez, el evento crea espacios y actividades para familias los fines de semana.

Por otro lado, aunque la carrera de curadoría es reciente en la ciudad — solo hay formación con talleres. Se mencionan nombres como Emiliano Valdés, Óscar Roldán, Martha Lucía Villafañe, Nydia Gutiérrez, Tony Evanko, Alberto Sierra y Lucrecia Piedrahíta, investigadores que, para presentar colecciones, organizan procesos con un hilo conductor; la curaduría propende por generar ideas y exposiciones, publicaciones, textos, proyectos. Harold Ortiz, explica que los curadores buscan proyectos y crean diálogo entre artistas y contexto. Gestionan el proyecto, consiguen financiación y acompaña el proceso de la galería que vende. Tony Evanko y Manuela Velásquez coinciden en que los curadores juegan un rol de apoyo, son eslabón esencial del proceso cultural y proyectarse internacionalmente, son estratégicos en sí. Sin embargo, también hay quien considera que para los artistas, es un rol complejo de integrar; Belarmino Miranda considera que a veces, no importa el artista sino los curadores.

En otro panorama, están las acciones de gestión, de la Secretaría de Cultura, centradas en políticas y presupuestos, que son necesarias para sostener proyectos culturales, pero deben entender dónde están las dinámicas de expansión. El modelo de gestión de la cultura en Medellín está relacionado con el recurso público y adopta problemáticas públicas relacionadas con equidad, formación, ciudadanía. Pero para el sector de artes plásticas y visuales no hay programas o políticas enrutados, y las agendas de divulgación según los públicos participantes, es dispersa. 
La secretaría, además, tiene una función social: fortalecer y promocionar expresiones artísticas, culturales y de cultura ciudadana; pero al respecto, los artistas y público entrevistados dicen que encontrar la agenda cultural de la ciudad es difícil, y así, para los artistas no hay demanda, o no hay forma de promocionar y hacer publicidad para comunicar la agenda cultural; mientras que para los públicos, no hay oferta. En todo caso, hay quien recalca que las mediciones son en taquillas y asistencia, no sobre públicos e intereses, que digan cómo acceden a productos culturales y qué buscan, lo cual puede ser estratégico.

El papel del Estado, destaca en convocatorias que relacionan recursos públicos con la creación de artistas y para propender el fortalecimiento del sector. Pero Harold Ortiz considera que otro rol de las instituciones públicas es el de formación de públicos, que aporta a la identidad cultural. Manuela Velásquez, coincide al decir que museos y Ministerio de Educación deberían encargarse de esa formación desde el sistema educativo y trabajar el tema cultural transversalmente. En resumen, hay un compromiso del gobierno con la difusión del arte como responsabilidad social, la promoción internacional y el apoyo a empresas de arte, pero recalcan sobre el tema de gratuidad, está bien apostarle a la subvención, pero separándola de la formación de públicos.

El siguiente componente es el de las instituciones educativas, la Universidad de Antioquia, la Universidad Nacional de Colombia y la fundación Universitaria Bellas Artes, ofrecen carrera de artes plásticas. Hay programas de posgrado y aparecen en escena los curadores formados, hay crítica y vitalidad en la producción de artistas jóvenes, pero en Medellín hay tanto artistas profesionales, como aficionados, el panorama puede ser emergente, pero también está en crecimiento.

Según Harold Ortiz, la exigencia académica es alta, pero como artistas necesitan preparación para desempeñarse profesionalmente y considerando el mercado.

Luego está la Empresa privada, que se relaciona con las bienales impulsadas por grandes empresas antioqueñas, que, además, son coleccionistas y mecenas que patrocinan la actividad artística y cultural de la ciudad. Las mayores colecciones corporativas del país pertenecen a empresas del Grupo Empresarial Antioqueño y las obras han trascendido la decoración para estar en museos, donde cada colección es pretexto para movilizar la apreciación y el consumo de la cultura. Entre los participantes del estudio, entidades como EPM, SURA, Bancolombia, Argos y Nutresa también se mencionan como patrocinadores de eventos y dueñas de colecciones que siguen la vocación coleccionista de Nicanor Restrepo, empresario que empezó la práctica. Pero los expertos coinciden en que no es fácil, pues los patrocinios siempre se hacen en términos de negocios y no con trasfondo cultural, importa la cantidad de asistentes que reconozca la marca, pero estratégicamente hablando en conjunción con la ley de mecenazgo puede haber oportunidades, por lo que habría que centrarse en generar confianza para invertir en lo cultural, en sensibilizar y convencer al sector privado del papel de la cultura en la responsabilidad social corporativa y el desarrollo.

Hay también organismos independientes o alternativos, que se han sumado a la esfera artística. Para desarrollar y promocionar proyectos hay espacios independientes como Taller 7 un espacio de encuentro y discusión de temas artísticos en el Museo de Antioquia y Casa Tres Patios, una iniciativa que combina prácticas de formación y apropiación social, cuya inversión suele tener alcance internacional por actividades como las residencias curatoriales que propician la investigación en entornos. Estos espacios posibilitan mostrar trabajos, experimentar, ser espacio de creación o hacer residencias. Por su parte Timebag bajo el liderazgo de los curadores Carlos Uribe y Gabriel Mario Vélez y la artista María Luisa Vélez, creó Colombia N.O.W., una exhibición que apoya espacios alternativos de exhibición de obras y artistas de diferentes ciudades del país. Todos estos espacios además, tienen presencia estratégicamente internacional.

Por otra parte, hay colectivos, especialmente en barrios periféricos, que ante la ausencia de la gestión pública, se organizaron y llevaron a que surgieran iniciativas que propician sus propias acciones a través del arte. Iniciativas civiles como la de Casa Kolacho, en la comuna 13, que por medio del Graffitour, hacen un recorrido estético, histórico y político, en palabras de Ciro, uno de sus creadores, reconstruyen la historia de la ciudad en materia tanto estética, como política y muestra la vida cotidiana asumiendo una formación de públicos entre habitantes y turistas; un ejemplo de estrategia independiente que integra otros sectores.

Este tipo de entidades, ha concentrado esfuerzos en generar espacios físicos para la creación, que pueden llamarse propuestas culturales, escenarios artísticos o talleres y espacios de trabajo colectivo que en palabras de representantes de esos colectivos son lugares donde se centralizan acciones, lugares de coincidencias; donde pasan cosas que no se definen con 
exactitud, pero funcionan para el fomento cultural de forma alternativa, e integran artistas y aficionados o emprendedores culturales y además, son autogestionados. Se han convertido en un referente para el arte en Medellín, nacen a partir de las comunidades e incluso ofrecen residencias artísticas, hacen investigaciones, realizan exposiciones, subastas, publicaciones y proyectos curatoriales, son espacios de encuentro, mediación y circulación para varias manifestaciones culturales. Finalmente, surgieron entidades identificadas como de tipo independiente, entre las cuales surgen Corporación Arteunico, Colectivo Artel, La Casa Redonda, Por Estos Días, La Pascasia, Fundación BAT, Campos de Gutiérrez, Casa de las Estrategias-Casa Morada, Corporación Cultural Nuestra Gente, El Puente Lab, Platohedro, Proyecto NN, Rizoma Ultravioleta, UN/loquer, Plazarte, Colectivo Morada, Satélite Estación de Arte y Proyecto El Faire. Todas estas, proyectos con tonos y objetivos diferenciados, pero que coinciden en que están pensados como instancias de experimentación y de ensayo, como laboratorios en los que se cocinan ideas y talentos. Dan cuenta de nuevas formas de relación y movilidad para artistas locales y por el mundo. Mantienen la agenda viva, crean comunidad y, a su manera, complementan el trabajo de organizaciones e instituciones. Estratégicamente se muestran como una alternativa exitosa y dan a la ciudad un nuevo aire.

La experimentación de estos espacios traducida en producción de obras que salen al mercado se relaciona con la vocación, y por eso, el MDE les abrió puertas; según Tony Evanko, funcionan como puentes entre prácticas comunitarias no relacionadas con el arte contemporáneo, pero tienen un papel importante en la transformación de la ciudad, lo que estratégicamente, amplía la influencia del arte en general, a diversos públicos, le hace cercano.

\section{Estrategias para la gestión cultural hacia sus mercados}

En Medellín, es necesario hablar del factor diferenciador de la cultura -en términos de marketing estratégico- , para su gestión en general; por eso identificar acciones, políticas y procedimientos que se vienen ejecutando y funcionan, es una táctica relevante para su oferta, promoción e inversión. En la ciudad la industria cultural se mueve a nivel local y en varios ámbitos que estimulan la práctica y las manifestaciones culturales. Hay eventos y artistas que suman a las acciones para formar público, a crear espacios que conducen al arte y al hacer ciudad, MDE incluye temas académicos y de relacionamiento comercial, involucra artistas nacionales e internacionales, curaduría de renombre, colectivos y espacios autogestionados; además, comprendiendo políticas locales de promoción del arte. Harold Ortiz destaca El Timebag Art Show, un encuentro de artistas reconocidos e independientes de arte contemporáneo, que integra modelos de gestión con una dinámica que construye cultura artística, reuniendo a públicos, aficionados y coleccionistas. Mostrando que no todo puede venir desde la institucionalidad, pues permite establecer conversación con empresas, encontrar patrocinios y fortalecer canales de promoción. También se acercan a los medios para contar historias o dejar que el arte ocurra y acercarse a los públicos. Esta dinámica de corte cultural, podría centrarse en generar beneficios económicos para todos los actores y, por ende, para la ciudad.

En cuanto a las acciones de las instituciones, para varias personas entrevistadas, los recursos estatales, no son visibles, están concentrados en los museos, o consideran que no se generan beneficios económicos, que la rentabilidad es "meramente social". Lo que se reitera al ver recursos que se centran en el fortalecimiento de museos, programas de entrada libre y apoyos concertados. Cuando hay inversiones mayores como la del MAMM, vienen de fuentes institucionales y de aportes de entidades como Fundación Sofía Pérez de Soto, Bancolombia, Sura, Fundación Fraternidad Medellín, Conconcreto, Argos y Ministerio de Cultura. Pero la inversión en cultura y la asignación presupuestal siempre se enfrentan al recorte, aun cuando en 2019, el presupuesto de cultura en Medellín estuvo en su punto histórico más alto y el presupuesto para 2020 es de más de 87 mil millones, un aumento presupuestal reajustado que luego se encontró con la situación originada por la pandemia y llevó a una crisis del sector.

Entonces, qué factores se deberían tener en cuenta para el fortalecimiento del ecosistema de artes plásticas y visuales. Hasta ahora, las convocatorias han sido mecanismo de fortalecimiento e impulso a creadores y artistas y si bien el soporte institucional del sector público a museos o eventos como los salones regionales o los encuentros MDE, activan el sector, según Carlos Bermúdez, artista, esta dependencia peligra en continuidad de acuerdo con los cambios de gobierno. Por lo que hay que encontrar oportunidad en la producción y comercialización del arte local e identificar el ecosistema, esto, facilitaría la apropiación de procesos e identificarse para un trabajo mancomunado que integre las estrategias de los colectivos, que comenzaron a crear un mundo propio, para experimentar en compañía, dando entrada a los espacios autogestionados. 
Pensarse como parte de un ecosistema para gestionar y comercializar la producción cultural considerando todo lo que al interior de este sucede, permitiría mover la obra de artistas, no solo a través de los galeristas, invitaría a los artistas que venden lo que producen en sus talleres, que realizan ventas en espacios informales en su propio círculo, en tiendas de diseño o por perfiles de redes sociales a ser parte de ese todo, pero esto requiere de comunicación estratégica. Con relación a lo anterior, Harold Ortiz, explica que para la buena oferta no hay mercado y demanda a nivel local, porque está por desarrollarse, y por eso se recurre a la venta online, o salir del país. Esa dinámica con nuevos intermediarios y canales acercando obras y artistas a nuevos mercados, necesita de formación y práctica en el uso de canales digitales, para llegar a las personas en redes sociales, pero poco o nada saben de las artes plásticas de la ciudad según Belarmino Miranda y eso implica hacer estrategias de comunicación promocional.

Frente a la formación de público, hay que considerar la demanda de consumo cultural en Medellín, que según la encuesta de percepción ciudadana Medellín Cómo Vamos $^{3}$, es para cine, ferias, conciertos, literatura, teatro, museos, sitios históricos, festivales o tertulias. Los hábitos de consumo del mercado del arte no le hacen dinámico cuando los públicos no están formados para consumir expresiones artísticas y mucho menos compra de obras, y aunque la ciudad ha promovido el arte y la cultura, subvencionando eventos, eso hace que piensen que debe ser gratuito, dificultando más atraer público que no invierte en cultura. Allí hay que retomar estrategias como Lokkus o Plecto, galerías que, según Manuela Velásquez, quieren trascender esa oposición entre experimentación, producción y venta, mediante el acompañamiento a los artistas para acercarlas a un público de compradores locales.

El diferencial del mercado de artes plásticas y visuales en Medellín es que es social y público, que los mismos artistas, están generando y gestionando espacios. El mercado viene orientándose a lugares alternativos y creativos, donde los artistas se unen, porque son eclécticos y comprensivos con otras disciplinas como la música, la literatura y las audiovisuales y recurren a la autogestión. Para María Mercedes González, la oferta en la ciudad es amplia y variada y cuenta con una infraestructura de primer nivel — parques culturales, museos y universidades. Tambien entre las personas entrevistadas, se recalca un trabajo colectivo para compartir lo que se

3 https://tinyurl.com/jtstd2x8 hace en distintos lugares y cada institución se esfuerza por mostrar lo qué hace; pero reiteran la necesidad de una estrategia de comunicación para una agenda, ofertando las distintas posibilidades y lugares. En aspectos promocionales la secretaría de Cultura creó una publicación propia y centralizada como guía para artistas, habitantes y visitantes y creó el portal de la agenda cultural de Medellín, pero tienen mayor impacto inciativas como Catalyst, hecha por residentes extranjeros.

Finalmente, algo que inhibe el desarrollo del mercado para este sector, es que la agenda se concentra en periodos, mientras la oferta en enero es pobre, en agosto o septiembre hay varias opciones diarias.

\section{Discusión y Conclusiones}

Las evidencias permiten identificar oportunidades estratégicas para la formación de públicos como base para el desarrollo y construcción de la demanda y el consumo cultural para este sector, a partir de propiciar conocimiento en temas artísticos, que motivan a asistir y a consumir obras y eventos culturales, pero donde hay que explorar factores de interés y de motivación, y de percepción e intención en términos de segmentación, como plantean Gürel y Nielsen (2019).

Desde el componente académico, hay que implementar cátedras de desarrollo de negociación y difusión o marketing cultural, para que futuros artistas obtengan herramientas, recursos y capacidades para relacionarse con consumidores y ser canal directo con el mercado, aplicando estrategias propias y generando ventajas en el diseño y control de comunicaciones con personalización de mensajes. Esto contribuiría al desarrollo de públicos y audiencias, pues partiría de un conocimiento de sus propios públicos, al trabajar según cada segmento y definir su posicionamiento (Boorsma \& Chiaravalloti, 2010; Fernández \& Heikkilä, 2011).

Los museos como El MAMM y el Museo de Antioquia, vienen trabajando con efectos en los públicos, mediante varios programas, pero es necesario encontrar o afianzar lo realizado para llegar a personas sin ningún tipo de conocimiento, - considerando que el arte puede llegar a requerir explicaciones-, y generar en los públicos una interpretación que les lleve al disfrute y apropiación de las obras de arte, ojalá entendiéndolo como un derecho fundamental relacionado con el crecimiento personal, como lo plantea UNESCO 
(2012) o como patrimonio para todos (Silverman \& Fairchild-Ruggles, 2007).

Donde hay riqueza cultural el papel institucional y las políticas públicas son esenciales, pero es misión de todo el ecosistema de artes plásticas y visuales, integrar lo cultural y lo socioeconómico. Aunque hay presencia de actividades, programas, proyectos culturales y artísticos que apuntan a mejorar la visibilidad para propiciar accesibilidad al público y vincularse con visitantes, medios, patrocinadores y organizaciones, es necesario articular esfuerzos de gestión, con la coordinación entre entidades, a manera de mezcla de marketing y así poner en contacto productos y servicios con sus consumidores y públicos (Azuela Flores et al., 2010). El esquema de voz a voz (por referencia directa) es el canal de comunicación más utilizado en el medio, pero la difusión puede ser más extensiva, por eso, es importante estructurar una comunicación estratégica de marketing (Boorsma \& Chiaravalloti, 2010), que pueda suscribir alianzas y construir relaciones entre actores del ecosistema, para dar acceso a mayor cantidad de personas y canalizar la difusión de información. Esto implica propiciar trabajo colaborativo entre Academia, Estado y organizaciones culturales, articular la comunicación de la oferta cultural en la ciudad, de manera que los públicos y consumidores puedan tomar decisiones para consumir actividades artísticas (Boorsma, 2006).

Es necesario dejar en claro que un panorama general de eventos sin manejo estratégico cruza actividades relevantes económicamente, y puede desplazar eventos artísticos con menos trayectoria; por lo que habría que formalizar alianzas para permitir programar asistencias y generar tráfico siguiendo lo planteado por Pérez y Sánchez (2011). En ese marco, también se puede pensar el ecosistema de artes plásticas y visuales como estrategia de turismo que promueve la ciudad ante visitantes y otros públicos internacionales interesados en la ciudad. Los eventos y actividades que puedan ofrecerse son atractivo y fuente de toma de decisiones para conocer un territorio, su cultura y lo que sucede en la sociedad; por eso una estrategia es identificar zonas que albergan artistas y actividades que atraen públicos, generar dinámicas que posicionen lugares de la ciudad (Galí-Espelt, Majó-Fernández y Vidal-Casellas, 2000).

Ese fortalecer entornos desarrollando sinergias y gestión en pro del mercado cultural, implica generar directorios de artistas, - algo similar a guías turísticas-, permitiendo conocerles, divulgar sus obras, técnicas y ser excusa de encuentro. Puede considerarse un acelerador de emprendimientos culturales, incluyendo consultorías, para mejorar visibilidad y sacar el máximo partido de sus carreras, agregando así el valor agregado de la experiencia (Fernández \& Heikkilä, 2011; Gürel \& Nielsen, 2019). Hay que tener en cuenta también el valor percibido para el consumo cultural, que se manifiesta con la apropiación de la oferta cultural de experiencias muy bien valoradas, pero requiere propiciar una experiencia de consumo del arte percibida como inversión (Boix-Domenech y Lazzeretti, 2012).

Con lo anterior en cuenta, el marketing del arte, enfatiza la oferta y demanda, la decisión de consumo, o la promoción (Boorsma, 2006), pero es solo una parte de la gestión cultural, hace falta conectar a todos los actores del ecosistema. Puede trabajarse el mecenazgo con empresas privadas y financiar proyectos culturales a cambio de beneficios impositivos e incentivar la inversión en obras de arte. Estas transacciones se han realizado a través de fundaciones y organizaciones sin ánimo de lucro, pero también pueden trabajarse políticas orientadas al desarrollo y distribución de recursos para iniciativas culturales.

El tema cultural en Medellín ofrece un panorama amplio para futuras investigaciones, sobre factores motivantes de consumo cultural, diversificar usos del tiempo libre hacia actividades culturales, analizar la agenda cultural y divulgación de actividades, plantear la optimización de recursos públicos destinados para un mayor desarrollo, entender el funcionamiento de programas de formación de públicos, analizar el funcionamiento y propósito de las entidades autónomas o independientes o colectivos que actúan con iniciativas orientadas a la producción cultural o definir necesidades del ecosistema para organizar gestión cultural en otras ciudades a nivel nacional, o desde las actividades de otras entidades y desde políticas públicas.

Por otro lado, en cuanto al flujo del ecosistema de artes plásticas y visuales en Medellín, se puede analizar la distribución de información, documentar la estrategia de la cultura en general, investigar el rol de grandes empresas como patrocinadoras, hacer diagnósticos de las estrategias desde todas las ICC, identificar hábitos de consumidores comprometidos con la cultura, establecer el peso de del sector en la economía local y nacional, e identificar la influencia de la producción cultural en las dinámicas sociales y culturales de la región.

Este estudio tuvo una serie de limitaciones relacionadas con el acceso a personas expertas en el sector de 
artes, pero se logró hacer una entrevista por cada tipo de componente, esto puede llevar a pensar en un estudio adicional con más actores que refleje la postura de todo el ecosistema de artes con todos o hacerlo desde la perspectiva de los consumidores y públicos.

\section{Reconocimientos}

Este artículo hace parte del proyecto La oferta cultural y la experiencia turística en Medellín (767-000081), financiado por la Universidad EAFIT.

\section{Referencias}

Azuela Flores, J. I., Sanzo Pérez, M. J., \& Frernández Blanco, V. (2010). El marketing de la cultura y las artes: una evolución. Revista Nacional de Administración, 1(1), 23-36.

Boix Domenech, R., \& Lazzeretti, L. (2012). Las industrias creativas en España: una panorámica. Investigaciones Regionales: Journal of Regional Research, ISSN 1695-7253, 22, 181-206.

Boorsma, M. (2006). A strategic logic for arts marketing: Integrating customer value and artistic objectives. International Journal of Cultural Policy, 12(1), 73-92.

Boorsma, M., \& Chiaravalloti, F. (2010). Arts Marketing Performance: An Artistic-Mission-Led Approach to Evaluation. The Journal of Arts Management, Law, and Society, 40(4), 297-317.

Catalán Romero, S., \& González Rueda, A. J. (2014). Manual Atalaya de apoyo a la gestión cultural. In Atalaya: Vol. online. Atalaya. https://tinyurl.com/scy5nfms

Concejo de Medellín. (2016). Medellín primera ciudad con política pública de museos. Boletín 258.

Fernández, C. J., \& Heikkilä, R. (2011). El debate sobre el omnivorismo cultural. Una aproximación a nuevas tendencias en sociología del consumo. Revista Internacional de Sociología, 69(3), 585-606.

Geertz, C. (2003). La interpretación de las culturas. In Basic Books.
Gomez, J. (2006). Confluencias entre arte y ciudad. I Congreso Internacional de Arte y Entorno. La Ciudad Sentida. Arte, Entorno y Sostenibilidad. Próxima Parada: Berlín-Valencia, La ciudad(Berlín-Valencia).

González-Vélez, E. (2015). El ecosistema de las industrias culturales en Colombia. Revista UIS Humanidades, 41(2).

Guba, E., \& Lincoln, Y. (1994). Competing Paradigms in Qualitative Research. 105-117.

Gürel, E., \& Nielsen, A. (2019). Art museum visitor segments: Evidence from Italy on omnivores and highbrow univores. International Journal of Arts Management, 21(2), 55-69. Obtenido de: https://tinyurl.com/pffp434z

Kolb, B. (2013). Marketing for cultural organizations. In Oak Tree, Cork. Taylor \& Francis.

Ley 1834. (23 de mayo de 2017). Diario Oficial de la República de Colombia. No. 50.242. Bogotá D.C.

Ley 1943. (28 de diciembre de 2018). Diario Oficial de la República de Colombia. No. 50.820. Bogotá D.C.

López-Sintas, J. (2015). La construcción social de la experiencia cultural. In OmniaScience (Vol. 1).

Miller, T. (2009). From Creative To Cultural Industries. Cultural Studies, 23(1), 88-99.

Mollá Descals, A., \& Cuadrado García, M. (2000). La relación consumidor-artes: un equilibrio entre satisfacción de necesidades y libertad creativa. Estudios Sobre Consumo, ISSN-e 0212-9469, 53, 23-32.

O'Connor, J. (2000). The definition of the "Cultural Industries." The European Journal of Arts Education, 2, 15-27.

OEI. (2019). Economía Creativa. Boletin Informativo OEI. https://tinyurl.com/rt8rtx6d

Páez, Á., Atencio, E., \& Neüman, M. I. (2013). Categorías cibergráficas para el estudio de plataformas de gobierno electrónico*. 10(2), 247-262.

Pérez-Calero Sánchez, L., Pérez, L., \& Sánchez, C. (2011). Mercado Del Arte E Intermediarios: Una 
Perspectiva Actual * Art Market and Intermediaries: a Current Perspective. 23(1996), 537-550.

Memorias y patrimonio de Medellín (2015). Política pública de Museos del Municipio de Medellín, 1, Medellín.

Decreto 1935. (18 de octubre de 2018). Diario Oficial de la República de Colombia. No. 50.674. Bogotá D.C. Obtenido de: https://tinyurl.com/2wpuzj6t

Silverman, H., \& Fairchild-Ruggles, D. (2007). Cultural Heritage and Human Rights (H. Silverman \& D. FairchildRuggles (eds.)). Springer. https://doi.org/1

UNCTAD. (2014). Empowering Women Entrepreneurs through Information and Communications Technologies.

UNESCO. (2009). Sobre definiciones: ¿Qué se entiende por industrias culturales y creativas? / Organización de las Naciones Unidas para la Educación, la Ciencia y la Cultura. UNESCO. Obtenido de: https://tinyurl.com/4dkppxk5

UNESCO. (2012). Fácil guía 1: Cultura y nuestros derechos culturales - UNESCO Biblioteca Digital. Obtenido de: https://tinyurl.com/4ekfabmv

Vattese, A. (2002). Invertir en arte : producción, promoción y mercado del arte contemporáneo. Ediciones Pirámide.

Vesga, O. M. (2005). La tradición en la enseñanza de las artes plásticas Tradition in Plastic Arts Teaching. El Artista, 2(1794-8614), 19-27. 